\title{
Reasons, Explanations and Decisions: Guidelines for Critical Thinking by Thomas McKay
}

Belmont, CA, USA: Wadsworth/Thomson Learning, 2000. pp. xx, 1-251. ISBN 0-534-57411-4. Paperbound \$53.95 USD.

\section{Daniel N. Boone}

In Reasons, Explanations and Decisions, Thomas McKay gives us an interesting addition to basic critical thinking textbooks. In the first half of the book, he offers a standard treatment of typical subjects (validity and soundness, premise and conclusion identification, vagueness and ambiguity, informal fallacies, analogy, etc.). The second half of the book, however, focuses on the nature of explanations and how best to evaluate them; on causal arguments and explanations, including experiments and statistical studies; and on decision-making. While other critical thinking texts may address the second set of topics briefly, McKay's text gives a substantial and detailed account of these subjects. Perhaps the closest comparison at the introductory level is with Ronald N. Giere's Understanding Scientific Reasoning (Harcourt Brace), which also takes a long look at statistical explanation, causal reasoning, and decisionmaking. The main difference is that Giere's book covers exclusively scientific reasoning, while McKay's, as already noted, devotes at least half the chapters to standard critical thinking topics. Thus, the book gives instructors a ready combination of the usual critical thinking topics with the more specialized inductive and decision theory topics.

Though McKay takes for the most part a standard approach to critical thinking areas in the first five chapters, he does introduce some novel elements. His presentation and discussion of "should" conclusions in Chapter 2 is thorough and quite good, and is a topic usually ignored by other texts. In Chapter 3 , he devotes more attention than usual to how context may affect meaning and reference. Chapter 3 also contains a delightful treatment of technical jargon and how to deal with it. Chapter 5, while short, is devoted to the proper uses and misuses of analogical reasoning and is innovative in its attempt to give a more dialectical account of analogies. Analogies, in their ideal uses for McKay, are "merely a way of making [relevant general principles] clear and vivid ..." (p. 105). The general principle, once clarified, will then be the basis of further inferences, and should be critically evaluated independently of the analogy.

Chapters 6 through 8 focus on explanations, with extra attention to causal explanations. This lengthy treatment is part of what sets this book off from other critical thinking texts, and, together with Chapter 9 on hypothesis test- 
ing and experimentation, provides an excellent introduction to inductive logic concepts and scientific reasoning. Chapter 6 introduces basic forms of explanation, such as appeals to generalizations, teleological explanations, and causal explanations. Chapter 7 discusses the nature of an inference to the best explanation, and then proceeds to address theoretical explanations, in both cases considering scientific and non-scientific cases. There is a strong emphasis on evaluation and criticism by appeal to criteria such as testability, scope, economy, consistency with other theories, lack of falsification, and absence of credible alternatives. Chapter 8 deals with arguments that generalize and arguments drawing causal conclusions, and methods of criticism for both. It further considers a number of problems or fallacies encountered in the misuse of statistics.

In Chapter 9, McKay pulls together the ideas covered in Chapters 6 through 8 , including the concept of inference to the best explanation, to specifically address the nature of hypothesis testing for causal explanations. His presentation of the use of control groups and experimental groups and how these combine with predictions to test causal hypotheses is clear and sufficiently non-technical. He introduces ethical and other factors that apply to controlled experiments and contrasts such experiments with the nature of less than ideal statistical studies. Finally, Chapter 10 provides an excellent introduction to decision theory with the coverage of methods such as satisficing, multi-attribute utility theory, maximin and maximax, and the expected utility method. McKay also attends to special problems in decision making, such as creativity and social conformity, the problems in equating money with value, and game theory.

One potentially troubling aspect of the book is McKay's treatment of the concept of "cause." On pp. 121-123, he first introduces his novel analysis of a "cause" and then repeats it later, notably pp. 162-163 in connection with statistical correlations, and again on pp. 179-180 as part of the discussion of hypothesis testing. McKay begins by contrasting necessary and sufficient conditions and notes that causal claims can be confusing because they may express either necessary or sufficient conditions indiscriminately. He then states, "For something to be a cause, it must be a necessary part of some sufficient set of conditions" (pp. 121-122, his italics here and below). McKay glosses this with an example (as an aside, this example is rather violent and, unfortunately, similar violent examples are used elsewhere in the book. Some may find the use of such examples offensive). In brief, Alice killed Bernie with a gun. Her using a gun was "not necessary"-she could have used poison, an axe, or a car bomb-and it was also "not sufficient"- the gun had to be loaded and working properly, and Bernie was only three feet away, did not move out of the way, wasn't already dead, etc. McKay then states, "A whole set of conditions, taking all of these circumstances together, is causally sufficient for Bernie's death, and Alice's pointing the gun and pulling the trigger is 
a part of what makes this set sufficient; it is a necessary part of a sufficient set of conditions" (p. 122). More formally, McKay explains further, "A is a necessary part of a set of conditions ( $\mathrm{S}^{*}$ ), that is sufficient to cause $\mathrm{Z}$. It is a necessary part because without $A$ the set is not sufficient (i.e., $S\left[=S^{*}\right.$ without $A$ ] is not sufficient) to cause $Z$. So we can say that $A$ is a cause of $Z$. This idea seems to apply to all causal claims" (p. 122, my own bracketed elaboration of the meaning of "S").

McKay's definition of "cause" is possibly troublesome in two ways. First, it appears contrary to other parts of McKay's own discussion of necessary and sufficient conditions. Alice's pointing the gun and pulling the trigger, according to McKay, is "not necessary," on the one hand, but on the other hand is "necessary" as a part of a sufficient set of conditions. I'm not charging McKay with inconsistency - he does make it adequately clear that these are distinct senses in which a given condition is and is not "necessary." Rather, the problem is the threat of possible confusion, especially to students using the text. It is not clear that students will appreciate that there is a difference between cases in which we describe something as a causally necessary condition (to use McKay's example, being exposed to measles virus is a necessary condition for getting measles) and cases in which something is a causally necessary condition (e.g., using a gun to kill someone) in McKay's special sense of being "a necessary part of some sufficient set of conditions." McKay, while distinguishing the two senses, is not at pains to labor over the distinction, and thus invites this possible confusion. Second, this first concern raises a deeper worry. McKay may think his account sheds light on the general concept of "causal necessity." The statement quoted above-that his idea "seems to apply to all causal claims"-is indicative of such a deeper intention. Further, the reappearance of this account in the other two contexts mentioned above-justifying causal claims by statistical correlations and testing causal hypotheses - is similarly suggestive. However, such gains in the understanding of causality are questionable.

(1) McKay's special notion of necessity as being "a necessary part of some sufficient set of conditions" applies to all the conditions in the set. Thus, the condition in his example that Bernie is only three feet away is also a necessary part of the sufficient set of conditions. If we suppose Alice is a very bad shot and requires victims to be at such close range, then there may indeed be some kind of statistical generalization that applies idiosyncratically to Alice's homicidal efforts. Since more proficient shooters may vary widely in their respective "necessities" of proximity to the target, however, any such generalizations will be of little causal interest. In such gloomy cases, conceivably involving homicide trials or sociological investigations of homicidal violence, more interesting nomic relationships might be sought, such as the nature and sources of Alice's rage at Bernie, the ready accessibility of guns to intended perpetrators, and so on. The point being: McKay's account of "cause" seems 
to apply equally to any conditions within a sufficient set, from profound to trivially idiosyncratic, and thus fails to help us understand what scientists and others are trying to accomplish in developing causal laws and causal hypotheses.

(2) The main debate among philosophers of science about causality seems to divide along the lines of those who take a "regularity" approach and those who prefer a stronger approach involving some kind of necessity. The former approach views causal laws as nothing other than descriptive summaries of observed regularities (an approach represented by a number of philosophers from Hume onwards). The latter approach attempts to view laws as, for example, expressing necessary relationships between universals (Dretske), or expressing metaphysical necessities of some kind (Kripke and Putnam). There is also Ronald Giere's quasi-instrumentalist attempt to view laws as expressing human cognitive impositions on the world. What isn't at all clear is how McKay's account of "cause" would enter or push forward this debate. Overall, simply because it so greatly relies on the definition of a cause as a "necessary" part of a sufficient set of conditions, it would seem to be in the "necessitarian" camp. However, in both Chapters 8 and 9, McKay emphasizes that we often don't know all the conditions in a set of sufficient conditions. In that event, "We support our belief in a causal connection by noticing a strong statistical association; then we produce a causal hypothesis that explains that statistical association" (p. 163). Or, "When we do not know all of the other elements of $\mathrm{S}^{*}$, the set that is sufficient to bring about the effect under consideration, statistical correlations can be important in establishing causal relations" (p. 180). These statements within their accompanying contexts certainly give major nods of approval to a "regularity" account. Whichever camp McKay inclines toward, in neither case does it seem that the type of "necessity" he defines is satisfyingly robust or that the relationship between his sense of causal necessity and statistical regularities is adequately spelled out.

Perhaps this is taking McKay too much to task for what is, after all, a small part of the book. The text has much to offer instructors and students. It is well organized and clearly written, provides chapter summaries for each chapter, and is truly replete with practical and readily applicable examples and exercises. Students will find these welcome in improving their critical thinking and decision making abilities. McKay is adept at expressing complex concepts in simple, accessible terms. A web-site instructor's manual is available, which includes answers to exercises and instructional tips. Anyone wishing to include the range of topics mentioned in the first paragraph above is well advised to give consideration to this text. 